\section{Prevención en la transmisión de Coronavirus-19: ¿qué tan preparado está el personal de salud en Chile?}

\author{
MATÍAS TAPIA P., ${ }^{1, a}$, MAGDALENA MÉNDEZ $Z^{1, a}$, \\ WILLY SEGUEL ${ }^{1}$, CONSUELO ROBLES ${ }^{1, a}$, SEBASTIÁN HURTADO ${ }^{1}$, \\ RUBÉN ÁVILA ${ }^{2}$, CRISTÓBAL DAVANZO $^{2}$, GONZALO BARRERA ${ }^{2}$, \\ SUSANA SEARLE ${ }^{3}$, RODRIGO TEJOS 3 ,b,c
}

\section{Adherence to COVID-19 trasmission preven- tion measures among health care workers}

Background: COVID-19 is highly transmissible, thus requiring strict measures to prevent its propagation. Aim: To report a survey about self-reported adherence to recommendations aimed to reduce the transmission of COVID-19, among health care personnel. Material and Methods: A cross-sectional survey among health professionals about adherence to recommendations to prevent COVID-19 transmission was carried out in a public hospital in Chile. The survey had 11 questions and was developed using Delphi methodology, according to the recommendations of the World Health Organization and the Centers for Disease Control. Results: The survey was answered by 137 of 155 invited workers. Hand washing, use of personal protection equipment, use of the elbow or tissue to sneeze, out-of-hospital hand washing and exclusive use of the uniform in the hospital, had an adherence of over $90 \%$. The adherence to face touching avoidance during working hours, and face mask use in the public areas, was over $50 \%$. No statistical differences were observed between gender or professionals. Conclusions: The adherence reported by health care workers was adequate in most of the evaluated recommendations.

(Rev Med Chile 2020; 148: 1589-1597)

Key words: Chile; COVID-19; Guideline Adherence; Health Personnel.
'Escuela de Medicina, Pontificia Universidad Católica de Chile. Santiago, Chile.

${ }^{2}$ Hospital de la Florida, Servicio de Salud Metropolitano Sur-Oriente. Santiago, Chile.

${ }^{3}$ Sección de Cirugía Plástica y Reconstructiva, División de Cirugía, Escuela de Medicina, Pontificia Universidad Católica de

Chile. Santiago, Chile.

Interno/a de Medicina.

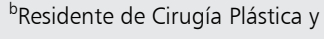
Reconstructiva.

'Magíster de Investigación en Ciencias de la Salud.

Trabajo no recibió financiamiento. Los autores declaran no tener conflictos de interés.

Recibido el 23 de junio de 2020, aceptado el 10 de noviembre de 2020.

Correspondencia a: Dr. Rodrigo Tejos Sufán Marcoleta 377, 2do Piso. Santiago, Chile. ratejos@uc.cl
L a enfermedad por coronavirus 2019 o "COVID-19" corresponde a la enfermedad viral causada por el SARS-CoV $2^{1,2}$. Este virus se encuentra en saliva, secreción nasofaríngea, sangre, lágrimas, orina, secreción bronquioalveolar y deposiciones, siendo sus principales vías de transmisión son las gotitas, fomites, contacto directo o estrecho, vía aérea y fecal, aunque ésta última no ha demostrado ser una vía relevante en la transmisión ${ }^{3}$.

El COVID-19 posee una elevada capacidad de transmisión desde el momento del contagio, incluso desde personas asintomáticas ${ }^{4}$. El período de incubación es de 2 a 14 días, sin embargo, la mayoría desarrolla síntomas antes del día 5-65. Del total de pacientes infectados, se estima que entre 40-50\% no desarrollarán síntomas ${ }^{6}$, o bien, mostrarán una enfermedad oligosintomática, cuya evolución es favorable en la mayoría de los casos ${ }^{7}$. Estas características han provocado la rápida propagación de la enfermedad, afectando hasta la fecha a más de 200 países $^{8}$.

En respuesta a la emergencia sanitaria global, la Organización Mundial de la Salud (OMS) decidió declarar el COVID-19 como pandemia el día 11 de marzo de $2020^{\circ}$. Dado las escasas herramientas 
terapéuticas, todo el esfuerzo se ha concentrado en disminuir su contagio entre personas ${ }^{10}$. Por esta razón, diversas organizaciones como la OMS, el centro de control y prevención de enfermedades de Estados Unidos (Centers for Disease Control and Prevention o CDC) y servicios de salud locales como el National Institute of Health (NIH) en Estados Unidos o el National Health Service (NHS) en Reino Unido han emitido diversas recomendaciones para disminuir la propagación del virus, tanto en ambiente hospitalario como extrahospitalario $^{7,11}$. Muchas de estas recomendaciones son basadas en la escasa evidencia disponible, en recomendaciones de expertos o son consideradas como buenas prácticas. Investigaciones previas han demostrado que la adherencia a las medidas preventivas y el uso correcto de elementos de protección personal (EPP) son fundamentales para evitar los contagios del personal de salud y de otros pacientes, por lo que deben ser fuertemente promovidas $^{10,12}$.

El objetivo de este trabajo es describir los resultados autorreportados por el personal sanitario sobre el conocimiento y adherencia de las recomendaciones para disminuir la transmisión del SARS CoV-2.

\section{Material y Métodos}

\section{Diseño y población de estudio}

Se realizó un estudio de tipo encuesta transversal. Se invitó a participar a todo el personal de salud disponible durante los días de aplicación de la encuesta en el servicio de Medicina Interna, Cirugía y Pabellones del Hospital Clínico de La Florida, Santiago, Chile.

\section{Encuesta}

Se realizó una búsqueda en la literatura sobre las medidas destinadas a la prevención de la diseminación del COVID 19 y se decidió tomar como base las recomendaciones referidas por la OMS,

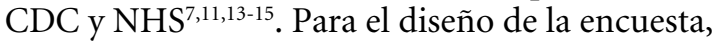
se elaboró un listado de aseveraciones con distintas formas de expresión de las recomendaciones. Posteriormente 3 miembros del equipo de trabajo (RT, RA y GB) analizaron y seleccionaron las preguntas a incluir en la encuesta mediante metodología Delfi modificada ${ }^{16}$. Esta encuesta consistió en 11 preguntas tipo Likert, donde los participantes seleccionaron la respuesta que más los representara (Anexo 1). Además, se incluyeron 5 preguntas adicionales sobre datos demográficos de la población.

\section{Responsabilidades éticas}

Este estudio fue aprobado por el comité de ética e investigación del Hospital de La Florida. Se realizó consentimiento informado a todos los participantes. Los datos fueron recolectados de forma anónima y confidencial.

\section{Trabajo de campo}

La encuesta fue realizada durante 3 días consecutivos del mes de abril de 2020. Dos miembros del equipo encuestaron a todo el personal de salud que aceptara participar. La encuesta se entregó en formato impreso y se dio un tiempo de 5 minutos para responder. La información se recolectó en sobres sellados que se depositaron en una caja recolectora para mantener la confidencialidad de las respuestas. Una vez concluido el período de trabajo de campo se abrieron los sobres y se realizó la transcripción de la información a una base de datos electrónica.

\section{Análisis estadístico}

Las variables demográficas se presentan como mediana/rango y número de participantes/ porcentaje, según correspondiera. Las respuestas de cada pregunta se expresan en número de respuestas/porcentaje. Se utilizó la prueba de test $\chi^{2}$ para realizar las comparaciones al estratificar según sexo y actividad laboral. Un valor $\mathrm{p}<0,05$ se consideró significativo.

Además, para evaluar la adherencia a las medidas, se creó la variable "Adherencia", correspondiente a la sumatoria de las respuestas muy frecuentemente y frecuentemente, expresadas como porcentajes del total de la población. En el caso de las preguntas 5 y 8 del cuestionario, la variable adherencia fue creada al sumar las respuestas nunca y casi nunca. Luego se definieron 3 categorías según el porcentaje obtenido en esta sumatoria: adherencia conforme (sumatoria $\geq 90 \%$ ) adherencia en vías de consolidación (sumatoria $89-50 \%$ ) y adherencia deficiente (sumatoria $\leq 49 \%$ ). El análisis estadístico se realizó utilizando software disponible comercialmente (SPSS 15 para Windows, Inc., Chicago, IL, EE. UU). 


\section{Resultados}

Se invitó a participar a un total de 155 funcionarios de la salud, de los cuales 137/88,4\% aceptaron ser entrevistados. Un total de 109/79,6\% sujetos de la muestra fueron mujeres y la mediana de edad fue de 29 años [21-55 años]. Aproximadamente la mitad de la población entrevistada fueron Técnicos en Enfermería Nivel Superior (TENS). Un total de 75 sujetos $(54,7 \%)$ utiliza el servicio de transporte público para ir al hospital. La Tabla 1 resume las características sociodemográficas de la población analizada.

\section{Medidas de prevención de transmisión intrahospitalaria de COVID-19 (MPI COVID-19)}

Las preguntas 1-6 corresponden a las MPI COVID-19 (Tabla 2). Al analizar las respuestas catalogadas como "muy frecuentemente", en cuanto al cumplimiento de medidas activas de prevención de transmisión del COVID-19, se observa que $92,7 \%$ reporta un adecuado uso del lavado de manos y $60,6 \%$ de los encuestados refieren buscar
Tabla 1. Características demográficas de la población analizada

\begin{tabular}{|lc|}
\hline Características demográficas & Total n = 137 \\
\hline Edad $^{+}$ & 29 años [21-55] \\
\hline Sexo femenino* & 109 / 79,6\% \\
\hline Actividad & \\
Auxiliares & $13 / 9,5 \%$ \\
Enfermeros/as & $34 / 24,8 \%$ \\
Internos/as de Medicina & $6 / 4,4 \%$ \\
Matrones & $10 / 7,3 \%$ \\
Médicos & $5 / 3,6 \%$ \\
TENS & $69 / 50,4 \%$ \\
Tipo de medio de transporte & \\
Privado & $62 / 45,3 \%$ \\
Público & $75 / 54,7 \%$ \\
Medio de transporte específico* & \\
Auto & $51 / 37,2 \%$ \\
Bicicleta & $2 / 1,5 \%$ \\
Pie & $9 / 6,6 \%$ \\
Metro, Transantiago, colectivos & $73 / 53,3 \%$ \\
Taxi, Uber, otros similares & $2 / 1,5 \%$ \\
\hline
\end{tabular}

${ }^{+}$Mediana/rango. *Número/porcentaje.

\section{Tabla 2. Frecuencias globales de respuesta sobre medidas de prevención de transmisión intrahospitalaria de COVID-19}

\begin{tabular}{|c|c|c|c|c|c|c|}
\hline n (\%) & $\begin{array}{l}\text { 1: Me lavc } \\
\text { Nunca } \\
0(0 \%)\end{array}$ & $\begin{array}{c}\text { manos antes } \\
\text { Casi Nunca } \\
0(0 \%)\end{array}$ & $\begin{array}{c}\text { espués de tener } \\
\text { Algunas Veces } \\
0(0 \%)\end{array}$ & $\begin{array}{c}\text { itacto con el pacie } \\
\text { Frecuentemente } \\
10(7,3 \%)\end{array}$ & $\begin{array}{l}\text { o su módulo de atenc } \\
\text { Muy Frecuentemente } \\
127(92,7 \%)\end{array}$ & $\begin{array}{l}\text { No Aplica } \\
0(0 \%)\end{array}$ \\
\hline \multicolumn{7}{|c|}{$\begin{array}{l}\text { Pregunta 2: Me esfuerzo por adquirir y utilizar correctamente los elementos de protección para prevención del contagi } \\
\text { y transmisión del COVID-19 (guantes, pechera, mascarilla, antiparras, otros) }\end{array}$} \\
\hline n (\%) & $\begin{array}{l}\text { Nunca } \\
1(0,7 \%)\end{array}$ & $\begin{array}{l}\text { Casi Nunca } \\
2(1,5 \%)\end{array}$ & $\begin{array}{l}\text { Algunas Veces } \\
\qquad 8(5,8 \%)\end{array}$ & $\begin{array}{l}\text { Frecuentemente } \\
\qquad 42(30,7 \%)\end{array}$ & $\begin{array}{l}\text { Muy Frecuentemente } \\
\qquad 83(60,6 \%)\end{array}$ & $\begin{array}{l}\text { No Aplica } \\
1(0,7 \%)\end{array}$ \\
\hline \multicolumn{7}{|c|}{ Pregunta 3: Considero necesario estornudar contra la fosa del codo o pañuelo y evito hacerlo sobre mis manos } \\
\hline n (\%) & $\begin{array}{c}\text { Nunca } \\
4(2,9 \%)\end{array}$ & $\begin{array}{l}\text { Casi Nunca } \\
1(0,7 \%)\end{array}$ & $\begin{array}{l}\text { Algunas Veces } \\
\quad 4(2,9 \%)\end{array}$ & $\begin{array}{l}\text { Frecuentemente } \\
19(13,9 \%)\end{array}$ & $\begin{array}{l}\text { Muy Frecuentemente } \\
109(79,6 \%)\end{array}$ & $\begin{array}{l}\text { No Aplica } \\
0(0 \%)\end{array}$ \\
\hline \multicolumn{7}{|c|}{$\begin{array}{l}\text { Pregunta 4: Realizo desinfección de instrumental una vez completada la atención de un paciente (Ej: fonendoscopio } \\
\text { esfigmomanómetro, oxímetro de pulso u otros) }\end{array}$} \\
\hline n (\%) & $\begin{array}{c}\text { Nunca } \\
4(2,9 \%)\end{array}$ & $\begin{array}{l}\text { Casi Nunca } \\
3(2,2 \%)\end{array}$ & $\begin{array}{l}\text { Algunas Veces } \\
12(8,8 \%)\end{array}$ & $\begin{array}{l}\text { Frecuentemente } \\
\qquad 40(29,2 \%)\end{array}$ & $\begin{array}{l}\text { Muy Frecuentemente } \\
75(54,7 \%)\end{array}$ & $\begin{array}{l}\text { No Aplica } \\
3(2,2 \%)\end{array}$ \\
\hline \multicolumn{7}{|c|}{ Pregunta 5: Durante un día normal de trabajo, me toco la cara sin darme cuenta } \\
\hline & Nunca & Casi Nunca & Algunas Veces & Frecuentemente & Muy Frecuentemente & No Aplica \\
\hline n (\%) & $3(2,2 \%)$ & $21(15,3 \%)$ & $63(46 \%)$ & $22(16,1 \%)$ & $28(20,4 \%)$ & $0(0 \%)$ \\
\hline \multicolumn{7}{|c|}{$\begin{array}{l}\text { Pregunta 6: Cuando he tenido contacto con un paciente sospechoso COVID-19, me esfuerzo por seguir el protocolc } \\
\text { establecido por mi centro de salud }\end{array}$} \\
\hline & Nunca & Casi Nunca & Algunas Veces & Frecuentemente & Muy Frecuentemente & No Aplica \\
\hline n (\%) & $7(5,1 \%)$ & $0(0 \%)$ & $3(2,2 \%)$ & $25(18,2 \%)$ & $87(63,5 \%)$ & $15(10,9 \%)$ \\
\hline
\end{tabular}


y utilizar correctamente los insumos de protección personal. Además, 79,6\% de los encuestados refiere estornudar en la fosa del codo/pañuelo y $54,7 \%$ realizan la desinfección del instrumental una vez completada la atención de un paciente. En relación a medidas de autocuidado, $36,5 \%$ de los encuestados refiere tocarse la cara muy frecuentemente o frecuentemente, mientras que solo $17,5 \%$ refiere realizar esta acción nunca o casi nunca. $\mathrm{Al}$ preguntar respecto al cumplimiento de protocolos de contacto con pacientes con COVID-19, se observa que $63,5 \%$ sigue muy frecuentemente el protocolo establecido por el centro de salud. Por otra parte, $5,1 \%(\mathrm{n}=7$ sujetos $)$ refirieron nunca seguir esta indicación. No se observaron diferencias estadísticamente significativas al estratificar por sexo ni por actividad laboral, en relación a las MPI COVID-19.

El análisis de adherencia a las MPI COVID-19 se presenta en la Figura 1. Se observa que las medidas consultadas en las preguntas 1 - 3 presentan un porcentaje de adherencia conforme ( $>90 \%)$, mientras que las medidas consultadas en las preguntas 4 y 6 presentan una adherencia en vías de consolidación, con un porcentaje de adherencia de $83,9 \%$ y $81,7 \%$, respectivamente. Destaca una adherencia deficiente en la pregunta 5 (recomendación sobre evitar tocar la región facial personal durante la jornada laboral), con un porcentaje de adherencia de un sólo 17,5\%.

\section{Medidas de prevención de transmisión extrahospitalaria de COVID-19 (MPE COVID-19)}

Las preguntas 7-11 corresponden a las MPE COVID-19 (Tabla 3). El 17,5\% de los encuestados considera haber recibido muy frecuentemente información respecto a medidas de prevención de contagio y transmisión del COVID-19 al llegar al hogar, mientras que 32,1\% declaró que nunca o casi nunca recibieron este tipo de información. $\mathrm{Al}$ consultar sobre las condiciones de uso del uniforme clínico, sólo 2,9\% $(\mathrm{n}=4)$ utiliza muy frecuentemente su uniforme tanto dentro como fuera del recinto hospitalario, mientras que $85,4 \%$ refiere que nunca debiera ser utilizado en ambos lugares. $\mathrm{Al}$ analizar las respuestas catalogadas como "muy frecuentemente" en cuanto al cumplimiento de medidas activas de prevención de transmisión del COVID-19, se observa que $75,2 \%$ de los encuestados se retira el calzado y uniforme de hospital en un lugar específico de su hogar y $100 \%$ lava sus manos al llegar al hogar. En relación al uso de mascarilla en la vía pública en ausencia de síntomas respiratorios, $12,4 \%$ de los encuestados declaró utilizarla muy frecuentemente, mientras que $60,6 \%$ declaró que nunca o casi nunca la utiliza. No se observaron diferencias estadísticamente significativas al estratificar por sexo ni por actividad laboral en relación a las MPE COVID-19.

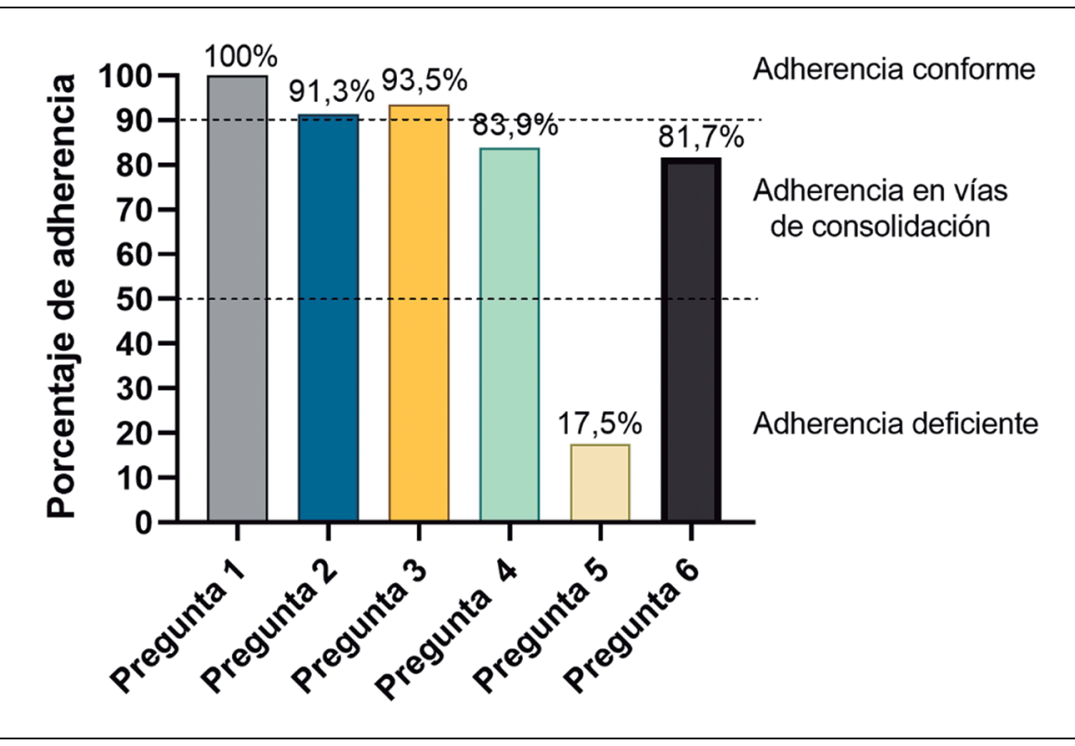

Figura 1. Análisis de adherencia a medidas de prevención de transmisión intrahospitalaria de COVID-19. 
Tabla 3. Frecuencias globales de respuestas sobre medidas de prevención de transmisión extrahospitalaria de COVID-19

Pregunta 7: El centro de salud en el cual me desempeño, me ha informado sobre las medidas de prevención de contagio y transmisión del COVID-19 al llegar a mi hogar

\begin{tabular}{|c|c|c|c|c|c|c|}
\hline & Nunca & Casi Nunca & Algunas Veces & Frecuentemente & Muy Frecuentemente & No Aplica \\
\hline n (\%) & $28(20,4 \%)$ & $16(11,7 \%)$ & $38(27,7 \%)$ & $31(22,6 \%)$ & $24(17,5 \%)$ & $0(0 \%)$ \\
\hline
\end{tabular}

Pregunta 8: Considero que el uniforme clínico debiese ser usado dentro y fuera del centro de salud

\begin{tabular}{|c|c|c|c|c|c|c|}
\hline n (\%) & $\begin{array}{c}\text { Nunca } \\
117(85,4 \%)\end{array}$ & $\begin{array}{c}\text { Casi Nunca } \\
9(6,6 \%)\end{array}$ & $\begin{array}{c}\text { Algunas Veces } \\
3(2,2 \%)\end{array}$ & $\begin{array}{c}\text { Frecuentemente } \\
4(2,9 \%)\end{array}$ & $\begin{array}{c}\text { Muy Frecuentemente } \\
\qquad 4(2,9 \%)\end{array}$ & $\begin{array}{c}\text { No Aplica } \\
0(0 \%)\end{array}$ \\
\hline
\end{tabular}

Pregunta 9: Me retiro el calzado y uniforme de hospital en un lugar específico de mi hogar para evitar recorrer mi casa con ellos puestos

\begin{tabular}{|c|c|c|c|c|c|c|}
\hline & Nunca & Casi Nunca & Algunas Veces & Frecuentemente & Muy Frecuentemente & No Aplica \\
\hline n (\%) & $8(5,8 \%)$ & $2(1,5 \%)$ & $4(2,9 \%)$ & $19(13,9 \%)$ & $103(75,2 \%)$ & $1(0,7 \%)$ \\
\hline
\end{tabular}

Pregunta 10: Lavo mis manos una vez que llego a mi hogar

\begin{tabular}{|c|c|c|c|c|c|}
\hline & Nunca & Casi Nunca & Algunas Veces & Frecuentemente & Muy Frecuentemente \\
\hline (\%) & $0(0 \%)$ & $0(0 \%)$ & $0(0 \%)$ & $3(2,2 \%)$ & $134(97,8 \%)$ \\
\hline
\end{tabular}

Pregunta 11: Utilizo mascarilla en la vía pública aún sin tener síntomas respiratorios

\begin{tabular}{|c|c|c|c|c|c|c|}
\hline n (\%) & $\begin{array}{c}\text { Nunca } \\
67(48,9 \%)\end{array}$ & $\begin{array}{l}\text { Casi Nunca } \\
16(11,7 \%)\end{array}$ & $\begin{array}{c}\text { Algunas Veces } \\
22(16,1 \%)\end{array}$ & $\begin{array}{c}\text { Frecuentemente } \\
15(10,9 \%)\end{array}$ & $\begin{array}{c}\text { Muy Frecuentemente } \\
17(12,4 \%)\end{array}$ & $\begin{array}{c}\text { No Aplica } \\
0(0 \%)\end{array}$ \\
\hline
\end{tabular}

El análisis de adherencia a las MPE COVID-19 se presenta en la Figura 2. Se observa que las medidas consultadas en las preguntas 8 y 10 presentan un porcentaje de adherencia conforme (>90\%), mientras que la medida consultada en la pregunta 9 presenta una adherencia en vías de consolidación, con un porcentaje de adherencia de $89,1 \%$.
Destaca además una adherencia deficiente en la percepción de los distintos profesionales sobre la información entregada por el centro de salud en relación a medidas de prevención de diseminación extrahospitalaria del COVID-19, así como sobre la recomendación de utilizar mascarilla en la vía pública aún sin presentar síntomas respiratorios,

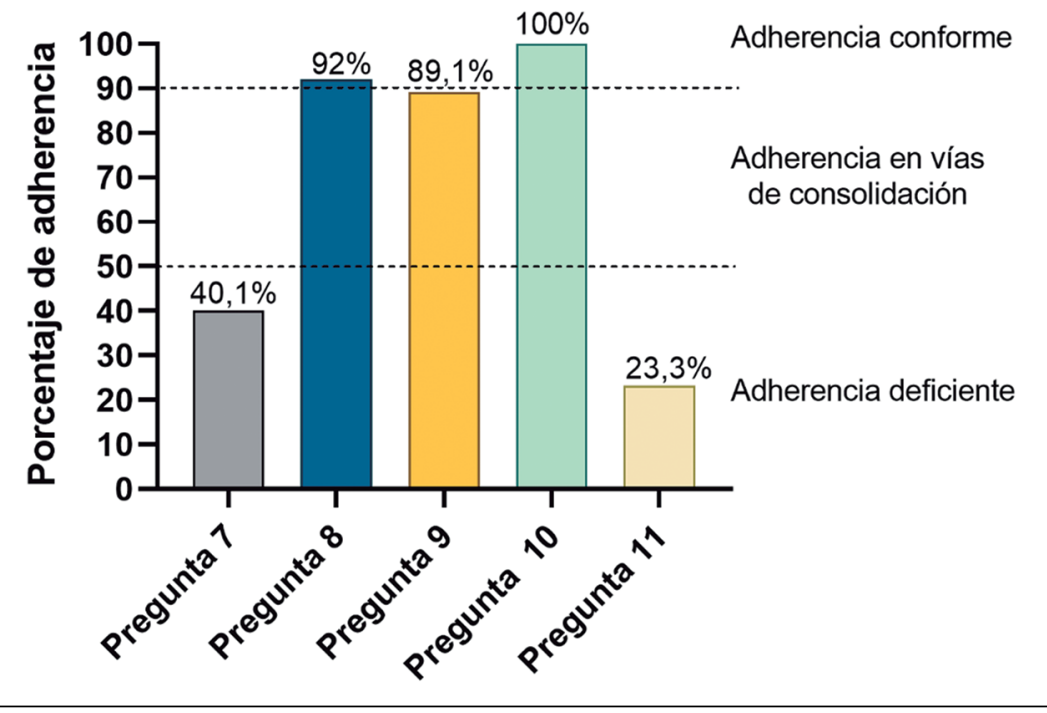

Figura 2. Análisis de adherencia a medidas de prevención de transmisión extrahospitalaria de COVID-19. 
con un porcentaje de adherencia de $40,1 \%$ y $23,3 \%$, respectivamente.

\section{Discusión}

En la actualidad existen limitadas alternativas terapéuticas para el tratamiento de la enfermedad causada por el COVID-19, por lo que la prevención de los contagios es primordial para el manejo epidemiológico. El personal de salud es un grupo particularmente expuesto a este tipo de infecciones, hecho que ha sido demostrado en pandemias previas. Para la epidemia del SARS en 2002 se reportaron aproximadamente 8.000 casos, de los cuales $21,07 \%$ (1.706) correspondieron a personal de salud ${ }^{17}$. En los años 2013-2015, durante la pandemia del Ébola, se describió un riesgo de contagio entre 21-32 veces mayor en el personal médico que en la población general ${ }^{3}$, produciéndose 880 casos en personal de salud, correspondiendo a $18,06 \%$ de los casos totales ${ }^{17}$. Para la pandemia actual por SARS CoV-2, hasta el 30 de abril, en relación a los datos disponibles sobre la Unión Europea, se han reportado aproximadamente 55.000 casos de COVID-19 en personal médico, correspondiente a $16,11 \%{ }^{17}$ del total de 340.000 casos hasta esa fecha en tal región, cifra que dista bastante de las estadísticas actualmente disponibles, con más de 35 millones de casos a nivel mundial. El personal de salud de primera línea tiene un elevado riesgo de infección por SARS CoV-2, con un HR de recibir un test positivo de 3,41 versus la población general $^{18}$. Por esta razón, es de especial relevancia la educación y autocuidado del personal de salud.

Uno de los principales hallazgos de este trabajo es la excelente adherencia al lavado de manos tanto dentro del recinto hospitalario, como al llegar al hogar. Esto es muy relevante ya que es una de las principales medidas de prevención para evitar contagios $^{19,20}$, razón por la cual ha sido fuertemente fomentada en el contexto de la pandemia por COVID-19. Por otra parte, diversos estudios observacionales internacionales muestran tasas de adherencia al lavado de manos en el personal de salud de entre $36,8 \%$ a $56,2 \%$, independiente del cargo que desempeñen en el servicio de salud ${ }^{21}$. Si bien los hallazgos de nuestra encuesta son prometedores, la información obtenida fueron datos auto-reportados y podría no representar la realidad clínica.
Una de las principales limitaciones del estudio es el menor porcentaje de médicos e internos de medicina encuestados $(8 \%)$ en comparación con estudios previos donde presentan entre $10-20 \%{ }^{22-24}$, ya que se sabe que este grupo corresponde a aquel con peor adherencia a las medidas de protección estándar y adicionales ${ }^{25,26}$. Sin embargo, esta diferencia probablemente se justifica por el menor número de médicos que asistía al hospital en período de pandemia debido a la existencia de un sistema de turnos, y el tiempo reducido que ellos permanecían en el hospital dada una limitación de exposición horaria del personal de salud, medidas adoptadas por la mayoría de los centros hospitalarios del país con el fin de disminuir la exposicion y contagios del personal médico.

Uno de los principales focos de atención debe ser mantener una correcta estrategia educativa para el personal de salud. Dentro de las medidas destinadas para favorecer una buena técnica y la adherencia al lavado de manos, la evidencia no es categórica. Una revisión sistemática del año $2017^{27}$, concluyó que la combinación de estrategias a multinivel, que involucran retroalimentación verbal, disponibilidad de soluciones de alcohol gel y recordatorios escritos o verbales, aumentan la adherencia al lavado de manos y podrían disminuir la tasa de infecciones intrahospitalarias.

La CDC recomienda como precauciones estándar (PE) el estornudar en la fosa del codo y la desinfección del instrumental clínico posterior al contacto con el paciente ${ }^{13,15}$. Si bien estas medidas tuvieron una alta adherencia reportada por la población estudiada, un alto porcentaje refiere tocarse la cara frecuentemente durante la labor clínica. Esto podría limitar el beneficio de lo anteriormente señalado, ya que expone al personal a adquirir la infección. La educación al personal de salud, la evaluación por pares y el uso de pautas o checklists han demostrado aumentar la adherencia a las $\mathrm{PE}^{28}$.

En relación a las MPE COVID-19 a través del uniforme clínico y el calzado, el personal encuestado tiene una buena adherencia a pesar de que las normativas actuales no hacen referencia a este hecho. Si bien la evidencia disponible no es categórica, muchos servicios de salud internacionales restringen el uso de indumentaria clínica exclusivamente al recinto hospitalario y recomiendan realizar su lavado o desinfección en el mismo hos- 
pital o en el domicilio particular de forma separada a las prendas cotidianas. Consideramos que esta medida debería ser promocionada y considerada como una buena práctica o standard of care basada en recomendaciones de expertos ${ }^{11}$.

El uso de mascarillas en personas asintomáticas ha tenido una gran discusión en el último tiempo. Al principio de la pandemia, la OMS hizo un llamado a reservar el uso de mascarillas quirúrgicas exclusivamente para pacientes sintomáticos o personas en riesgo de contagio (ej. personal de salud). Sin embargo, posteriormente esta medida fue extendida a personas asintomáticas en cuarentena que necesiten desplazarse de su hogar, así como a pacientes de alto riesgo de enfermedad severa ${ }^{29,30}$. El presente estudio fue realizado previo a que las autoridades sanitarias en Chile dictaminarán el uso obligatorio de mascarillas para transitar en la vía pública o en lugares con aglomeración de personas. Es decir, el uso de mascarilla reportado en nuestro estudio podría representar la realidad
Chilena previo a que se masificara esta práctica. Dada la evolución de la curva epidemiológica en nuestro país, consideramos que es posible haber obtenido un mayor beneficio si se hubiese aplicado de forma más precoz. Sin embargo, se requieren estudios diseñados específicamente para comprobar esta hipótesis.

\section{Conclusión}

La adherencia reportada por el personal de salud es adecuada para la mayoría de las recomendaciones de entidades internacionales para disminuir la transmisión del virus. Destaca una leve mayor adherencia a medidas de prevención de diseminación dentro del hospital que a medidas de diseminación extrahospitalaria. Es necesario potenciar las medidas deficientes para optimizar todos los recursos que disminuyan la diseminación de COVID-19.

\section{Anexo 1}

\section{ENCUESTA SOBRE MEDIDAS DE PREVENCIÓN DE DISEMINACIÓN EXTRAHOSPITALARIA DE LA INFECCIÓN POR SARS-COV 2 (COVID-19)}

\section{Introducción}

La siguiente encuesta tiene por objetivo analizar de forma cualitativa la puesta en práctica de diferentes prácticas que han demostrado disminuir la tasa de diseminación extrahospitalaria de infecciones, en el contexto de la pandemia mundial por el SARS-CoV 2, basándose en recomendaciones que la Organización Mundial de la Salud (OMS) ha hecho a los diferentes países. Esta encuesta consta de $\mathbf{5}$ preguntas sociodemográficas de respuesta corta y 10 afirmaciones, las cuales serán contestadas de forma anónima y confidencial.

¿Acepto participar de la encuesta? Sí, acepto No acepto

Sección I - aspectos sociodemográficos: rellene con respuesta corta donde corresponda

Edad: Sexo:

Ocupación:

Comuna de residencia:

Medio de transporte:

Sección II - afirmaciones: se le presentarán 10 afirmaciones sobre sus actividades dentro y fuera del hospital. Usted deberá marcar la casilla que más lo represente:

\begin{tabular}{|l|l|}
\hline Afirmación & Respuesta \\
\hline Me lavo las manos antes y después & $\square$ Nunca \\
de tener contacto con el paciente o & $\square$ Casi nunca \\
su módulo de atención & $\square$ Algunas veces \\
& $\square$ Frecuentemente \\
& $\square$ Muy frecuentemente \\
\hline
\end{tabular}

\begin{tabular}{|l|l|}
\hline Afirmación & Respuesta \\
\hline $\begin{array}{l}\text { Cuando he tenido contacto con un } \\
\text { paciente sospechoso para COVID 19, me } \\
\text { esfuerzo por seguir el protocolo } \\
\text { establecido por mi centro de salud. } \\
\text { (guantes, pechera, mascarilla y antiparras) }\end{array}$ & $\square$ Nunca \\
\hline
\end{tabular}




\section{Anexo 1 (continuación)}

\begin{tabular}{|c|c|c|c|}
\hline $\begin{array}{l}\text { Me esfuerzo por adquirir y utilizar } \\
\text { correctamente los elementos de } \\
\text { protección normados para } \\
\text { prevención del contagio y } \\
\text { transmisión del COVID } 19 \\
\text { (guantes, pechera, mascarilla y } \\
\text { antiparras) }\end{array}$ & $\begin{array}{ll}\square & \text { Nunca } \\
\square & \text { Casi nunca } \\
\square & \text { Algunas veces } \\
\square & \text { Frecuentemente } \\
\square & \text { Muy frecuentemente }\end{array}$ & $\begin{array}{l}\text { El Centro de Salud en el cual me } \\
\text { desempeño, me ha informado sobre las } \\
\text { medidas normadas para prevención de } \\
\text { contagio y transmisión del COVID } 19 \text { al } \\
\text { llegar a mi hogar }\end{array}$ & $\begin{array}{ll}\square & \text { Nunca } \\
\square & \text { Casi nunca } \\
\square & \text { Algunas veces } \\
\square & \text { Frecuentemente } \\
\square & \text { Muy frecuentemente }\end{array}$ \\
\hline $\begin{array}{l}\text { Considero necesario estornudar } \\
\text { contra la fosa del codo o pañuelo, y } \\
\text { evitar hacerlo sobre mis manos }\end{array}$ & $\begin{array}{ll}\square & \text { Nunca } \\
\square & \text { Casi nunca } \\
\square & \text { Algunas veces } \\
\square & \text { Frecuentemente } \\
\square & \text { Muy frecuentemente }\end{array}$ & $\begin{array}{l}\text { Considero que el uniforme clínico debiese } \\
\text { ser usado dentro y fuera del centro de } \\
\text { salud }\end{array}$ & $\begin{array}{ll}\square & \text { Nunca } \\
\square & \text { Casi nunca } \\
\square & \text { Algunas veces } \\
\square & \text { Frecuentemente } \\
\square & \text { Muy frecuentemente }\end{array}$ \\
\hline $\begin{array}{l}\text { Durante un día normal de trabajo, } \\
\text { me toco la cara sin darme cuenta }\end{array}$ & $\begin{array}{ll}\square & \text { Nunca } \\
\square & \text { Casi nunca } \\
\square & \text { Algunas veces } \\
\square & \text { Frecuentemente } \\
\square & \text { Muy frecuentemente }\end{array}$ & Lavo mis manos una vez llego a mi hogar & $\begin{array}{ll}\square & \text { Nunca } \\
\square & \text { Casi nunca } \\
\square & \text { Algunas veces } \\
\square & \text { Frecuentemente } \\
\square & \text { Muy frecuentemente }\end{array}$ \\
\hline \multirow[t]{2}{*}{$\begin{array}{l}\text { Realizo desinfección de } \\
\text { instrumental (ej. fonendoscopios, } \\
\text { termómetros, saturómetros) una } \\
\text { vez completada la atención de un } \\
\text { paciente }\end{array}$} & $\begin{array}{l}\square \text { Nunca } \\
\square \text { Casi nunca } \\
\square \text { Algunas veces } \\
\square \text { Frecuentemente } \\
\square \text { Muy frecuentemente }\end{array}$ & $\begin{array}{l}\text { Utilizo mascarilla en la vía pública aún sin } \\
\text { tener síntomas respiratorios }\end{array}$ & $\begin{array}{ll}\square & \text { Nunca } \\
\square & \text { Casi nunca } \\
\square & \text { Algunas veces } \\
\square & \text { Frecuentemente } \\
\square & \text { Muy frecuentemente }\end{array}$ \\
\hline & & $\begin{array}{l}\text { Me retiro el calzado y uniforme de hospital } \\
\text { en un lugar específico de mi hogar para } \\
\text { evitar recorrer mi casa con ellos puestos }\end{array}$ & $\begin{array}{ll}\square & \text { Nunca } \\
\square & \text { Casi nunca } \\
\square & \text { Algunas veces } \\
\square & \text { Frecuentemente } \\
\square & \text { Muy frecuentemente }\end{array}$ \\
\hline
\end{tabular}

\section{Referencias}

1. Guan W, Ni Z, Hu Y, Liang W, Ou C, He J, et al. Clinical Characteristics of Coronavirus Disease 2019 in China. N Engl J Med 2012; 382: 1708-20.

2. Yang X, Yu Y, Xu J, Shu H, Xia J, Liu H, et al. Clinical course and outcomes of critically ill patients with SARSCoV-2 pneumonia in Wuhan, China: a single-centered, retrospective, observational study. Lancet Respir Med 2020; 8: 475-81.

3. Li Q, Guan X, Wu P, Wang X, Zhou L, Tong Y, et al. Early Transmission Dynamics in Wuhan, China, of Novel Coronavirus-Infected Pneumonia. N Engl J Med 2020; 382(13): 1199-207.

4. Xie M, Chen Q. Insight into 2019 novel coronavirus An updated interim review and lessons from SARS-CoV and MERS-CoV. Int J Infect Dis. 2020; 94: 119-24.

5. Lauer S, Grantz K, Bi Q, Jones F, Zheng Q, Meredith $\mathrm{H}$, et al. The Incubation Period of Coronavirus Disease 2019 (COVID-19) From Publicly Reported Confirmed Cases: Estimation and Application. Ann Intern Med 2020; 172: 577-82.

6. Oran D, Topol E. Prevalence of Asymptomatic SARSCoV-2 Infection. Annals of Internal Medicine. 2020; 173 (5): 362-7.
7. Centers for Disease Control and Prevention. Interim Clinical Guidance for Management of Patients with Confirmed Coronavirus Disease (COVID-19); [updated 2 de junio de 2020; citado el 10 de junio de 2020] Available from: https://www.cdc.gov/coronavirus/2019-ncov/hcp/clinical-guidance-management-patients.html

8. Dong E, Du H, Gardner L. An interactive web-based dashboard to track COVID-19 in real time. Lancet Infect Dis 2020; 20: 533-4.

9. Cucinotta D, Vanelli M. WHO Declares COVID-19 a Pandemic. Acta Bio Med 2020; 91 (1): 157-60.

10. Centers for Disease Control and Prevention. How to Protect Yourself. Coronavirus Disease 2019 (COVID-19); [updated 24 de abril de 2020; citado el 29 de mayo de 2020]. Available from: https://www.cdc.gov/ coronavirus/2019-ncov/prevent-getting-sick/prevention. html

11. National Health Service (NHS). Uniforms and workwear: guidance for NHS employers. [updated 20 de abril de 2020; citado el 29 de mayo de 2020] Available from: https://www.england.nhs.uk/wp-content/ uploads/2020/04/Uniforms-and-Workwear-Guidance-2-April-2020.pdf

12. Verbeek J, Rajamaki B, Ijaz S, Mischke C, Ruotsalainen 
J, Edmond MB, et al. Personal protective equipment for preventing highly infectious diseases due to exposure to contaminated body fluids in healthcare staff (Review). Cochrane Database Syst Rev 2020; 7 (7): CD011621.

13. Centers for Disease Control and Prevention. Environmental Infection Control Guidelines. [updated 2019 Jul 23; cited 2020 May 29]. Available from: https://www. cdc.gov/infectioncontrol/guidelines/environmental/ index.html

14. Centers for Disease Control and Prevention. 2007 Guideline for Isolation Precautions: Preventing Transmission of Infectious Agents in Healthcare Settings. [updated 29 de julio de 2019; citado el 29 de mayo de 2020]. Available from: https://www.cdc.gov/infectioncontrol/ guidelines/isolation/index.html

15. Organización Mundial de la Salud. Prevención y control de infecciones durante la atención sanitaria de casos en los que se sospecha una infección por el nuevo coronavirus (nCoV). Orientaciones Provisionales. Orientaciones técnicas sobre el nuevo coronavirus (2019-nCoV). [updated 25 de enero de 2020; citado el 29 de mayo de 2020]. Available from: https://apps.who.int/iris/bitstream/handle/10665/330685/9789240001114-spa.pdf

16. Hasson F, Keeney S, McKenna H. Research guidelines for the Delphi survey technique. J Adv Nursing 2000; 32: 1008-115.

17. Xiao J, Fang M, Chen Q, He B. SARS, MERS and COVID-19 among healthcare workers: A narrative review. Journal of Infection and Public Health 2020; 13 (6): 843-8.

18. Nguyen L, Drew D, Graham M, Joshi A, Guo C, Ma W, et al. Risk of COVID-19 among front-line health-care workers and the general community: a prospective cohort study. The Lancet Public Health. 2020; 5 (9): e475-83.

19. Luong Thanh B, Laopaiboon M, Koh D, Sakunkoo P, Moe H. Behavioural interventions to promote workers' use of respiratory protective equipment. Cochrane Database Syst Rev 2016; 12 (12): CD010157.

20. World Health Organization. WHO guidelines on hand hygiene in health care. Geneva: World Health Organization; 2009. Available from: https://www.ncbi.nlm.nih. gov/books/NBK144013/

21. Randle J, Arthur A, Vaughan N, Wharrad H, Windle
R. An observational study of hand hygiene adherence following the introduction of an education intervention. J Infect Prev 2014; 15 (4): 142-7.

22. Vidal Gamboa C, Palavecino Sáez I, Moya Rivera P, Toro Huerta C, Hoffmeister Arce L. Calidad de Vida del Personal de Salud y su Relación con el Ausentismo. Ciencia \& Trabajo 2017; 19 (60): 188-93.

23. Ordenes N. Prevalencia de Burnout en trabajadores del hospital Roberto del Río. Rev Chil Pediatr 2020; 75 (5): 449-54.

24. Avendaño C, Bustos P, Espinoza P, García F, Pierart T. Burnout y Apoyo Social en Personal del Servicio de Psiquiatría de un Hospital Público. Ciencia y Enfermería 2009; 15 (2): 55-68.

25. Gammon J, Morgan-Samuel H, Gould D. A review of the evidence for suboptimal compliance of healthcare practitioners to standard/universal infection control precautions. J Clin Nurs 2008; 17 (2): 157-67.

26. Michalsen A, Delclos GL, Felknor SA, Davidson AL, Johnson PC, Vesley D, et al. Compliance with universal precautions among physicians. J Occup Environ Med 1997; 39 (2): 130-7.

27. Gould D, Moralejo D, Drey N, Chudleigh J, Taljaard $\mathrm{M}$. Interventions to improve hand hygiene compliance in patient care. Cochrane Database Syst Rev 2017; 9 (9): CD005186.

28. Moralejo D, El Dib R, Prata R, Barretti P, Corrêa I. Improving adherence to Standard Precautions for the control of healthcare-associated infections. Cochrane Database Syst Rev 2018; 2(2): CD010768.

29. Organización Mundial de la Salud. Consejos sobre la utilización de mascarillas en el entorno comunitario, en la atención domiciliaria y en centros de salud en el contexto del brote de nuevo coronavirus (2019-nCoV). Directrices provisionales. [updated 29 de enero de 2020; citado el 29 de mayo de 2020]. Available from: https:// apps.who.int/iris/bitstream/handle/10665/330999/ WHO-nCov-IPC_Masks-2020.1-spa.pdf.

30. World Health Organization. Advice on the use of masks in the context of COVID-19. Interim Guidance. [updated 5 de junio de 2020; citado el 10 de junio de 2020]. Available from: https://apps.who.int/iris/bitstream/ handle/10665/331693/WHO-2019-nCov-IPC_Masks2020.3-eng.pdf. 